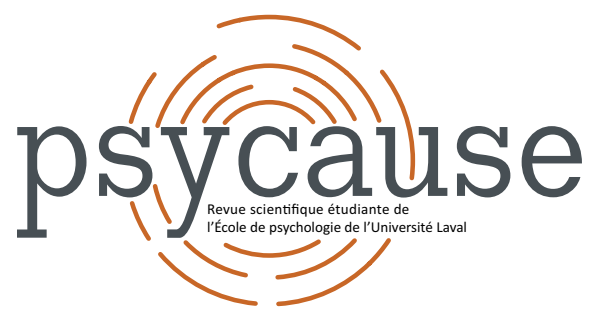

\title{
PSYCAUSE
}

Revue scientifique étudiante de l'École de psychologie de l'Université Laval

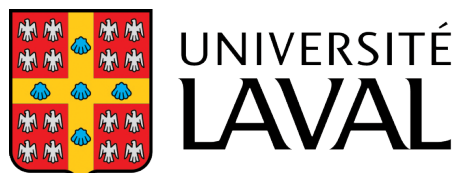

Faculté des sciences sociales École de psychologie

NOVEMBRE 2020 - VOL. $10 \mathrm{~N}^{\circ} 2$

\section{LA RELATION ENTRE LE PERFECTIONNISME ET LES SYMPTÔMES SOMATIQUES CHEZ LES FEMMES ATTEINTES D'UN CANCER DU SEIN}

\author{
Nakita SÉGUIN-KAERCHER ${ }^{1}$, Justine CADORETTE ${ }^{1}$, Véronique MASSICOTE ${ }^{1,2,3}$, Hans IVERS ${ }^{1,2,3}$ \& \\ Josée SAVARD ${ }^{1,2,3}$ \\ ${ }^{1}$ École de psychologie, Université Laval \\ ${ }^{2}$ Centre de recherche du CHU de Québec-Université Laval \\ ${ }^{3}$ Centre de recherche sur le cancer de l'Université Laval \\ *nakita.seguin-kaercher.1@ulaval.ca
}

\section{Pour citer l'article}

Séguin-Kaercher, N., Cadorette, J., Massicote, V., Ivers, H., \& Savard, J. (2020). La relation entre le perfectionnisme et les symptômes somatiques chez les femmes atteintes d'un cancer du sein. Psycause: Revue scientifique étudiante de l'École de psychologie de l'Université Laval, 10(2), 15-17. 
sentation de l'avatar et le genre du participant n'est pas significatif, donc aucune analyse d'effets simples n'a été effectuée. Une interprétation possible porte sur l'hypothèse des similitudes entre les sexes (Hyde, 2005). Les hommes et les femmes seraient similaires sur plusieurs habiletés sociales (p. ex., sociabilité, communication) et certains processus cognitifs (p. ex., raisonnement abstrait, moral), ce qui pourrait se généraliser à la perception de l'empathie. Les données obtenues dans la présente étude montrent l'importance d'une représentation d'avatar féminin pour augmenter l'empathie perçue chez un observateur. Elles permettront aussi d'améliorer les plateformes virtuelles existantes, en plus d'améliorer les connaissances sur les interactions empathiques humain-machine. Dès lors, il serait pertinent d'approfondir l'apport de chaque comportement non verbal (regard, posture et expression faciale) dans la perception de l'empathie exprimée par différents avatars (féminins/ masculins) en fonction du genre du participant.

\section{Références}

Brugel, S., Postma-Nilsenova, M., \& Tates, K. (2015). The link between perception of clinical empathy and nonverbal behavior: The effect of a doctor's gaze and body orientation. Patient Education Counseling, 98(10), 1260-1265. https:// doi.org/10.1016/j.pec.2015.08.007
Christov-Moore, L., Simpson, E. A., Coude, G., Grigaityte, K., lacoboni, M., \& Ferrari, P. F. (2014). Empathy: gender effects in brain and behavior. Neuroscience \& Biobehavioral Reviews, 46 Pt 4, 604-627. https://doi.org/10.1016/j. neubiorev.2014.09.001

Decety, J., \& Jackson, P. L. (2004). The functional architecture of human empathy. Behavioral and Cognitive Neuroscience Reviews 3(2), 71-100. https://doi. org/10.1177/1534582304267187

Doherty, R. W., Orimoto, L., Singelis, T. M., Hatfield, E., \& Hebb, J. (1995). Emotional Contagion: Gender and Occupational Differences. Psychology of Women Quarterly, 19(3), 355371. https://doi.org/10.1111/j.1471-6402.1995.tb00080.x

Hyde, J. S. (2005). The gender similarities hypothesis. American Psychologist, 60(6), 581-592. https://doi. org/10.1037/0003-066X.60.6.581

Hyde, J. S., Bigler, R. S., Joel, D., Tate, C. C., \& van Anders, S. M. (2019). The future of sex and gender in psychology: Five challenges to the gender binary. American Psychologist, 74(2), 171-193. https://doi.org/10.1037/amp0000307

Tepper, D. T., \& Haase, R. F. (1978). Verbal and nonverbal communication of facilitative conditions. Journal of Counseling Psychology, 25(1), 35-44. https://doi. org/10.1037/0022-0167.25.1.35

\title{
Pour citer l'article
}

Laverdière, R., Robichaud, L.-A., Toulouse-Fournier, A., Marcoux, A. \& Jackson, P. (2020). Influence du genre sur la perception de l'empathie à l'aide d'avatars. Psycause: Revue scientifique étudiante de l'École de psychologie de l'Université Laval, 10(2), 13-15.

\section{LA RELATION ENTRE LE PERFECTIONNISME ET LES SYMPTÔMES SOMATIQUES CHEZ LES FEMMES ATTEINTES D'UN CANCER DU SEIN}

\author{
Nakita SÉGUIN-KAERCHER ${ }^{1}$, Justine CADORETTE1 ${ }^{1}$, Véronique MASSICOTE1',2,3, Hans IVERS 1,2,3 \& Josée SAVARD ${ }^{1,2,3}$ \\ ${ }^{1}$ École de psychologie, Université Laval, ${ }^{2}$ Centre de recherche du CHU de Québec-Université Laval, ${ }^{3}$ Centre de recherche \\ sur le cancer de l'Université Laval \\ *nakita.seguin-kaercher.1@ulaval.ca
}

\section{Mots-clés : Cancer, cancer du sein, perfectionnisme, symptômes somatiques}

Le cancer du sein représente le deuxième diagnostic de cancer en importance dans la population canadienne en 2020 (Société canadienne du cancer, 2020). Cette maladie et son traitement sont associés au développement de plusieurs symptômes somatiques qui peuvent affecter le fonctionnement et la qualité de vie des patientes (Ferreira et coll., 2008). Il est donc pertinent d'explorer différents facteurs qui peuvent les influencer. Parmi ceux-ci, on retrouve certains traits de personnalité, tels que le perfectionnisme (Saboonchi \& Lundh, 2003).
Les aspirations perfectionnistes correspondent à l'entretien de normes personnelles élevées, souvent irréalistes, envers soi-même. Les préoccupations perfectionnistes se manifestent, quant à elles, par une autocritique sévère, des préoccupations excessives envers le jugement des autres et des réactions négatives face à l'échec (Sirois \& Molnar, 2016).

Le perfectionnisme est associé à certains effets négatifs chez les gens souffrant de maladies chroniques (Sirois \& Molnar, 2016). Quelques données de recherches suggèrent 
que le perfectionnisme est lié au profil symptomatique de conditions médicales chroniques telles que le syndrome de fatigue chronique (White \& Schweitzer, 2000). À ce jour, aucune étude n'a encore été réalisée dans le contexte du cancer du sein. L'objectif de cette étude est d'étudier l'association entre les deux dimensions du perfectionnisme (c.-à-d., aspirations perfectionnistes et préoccupations perfectionnistes) et les symptômes somatiques (fréquence et interférence perçue) sur les plans transversaux (objectif 1) et longitudinaux (objectif 2).

\section{Méthode}

La présente étude est réalisée à partir des données d'un projet de recherche de plus grande envergure (Savard, Ivers, Villa, Caplette-Gingras, \& Morin, 2011; Savard, Villa, Ivers, Simard, \& Morin, 2009). Dans le cadre du présent projet, les données de 459 femmes traitées pour un cancer du sein à L'Hôtel-Dieu de Québec et à l'Hôpital du Saint-Sacrement sont utilisées. Ces femmes, âgées entre 18 et 80 ans, devaient avoir reçu un diagnostic du cancer non métastatique et être sur le point de subir une chirurgie à visée curative. Cette étude longitudinale comprend six temps de mesure. Le premier temps de mesure a lieu durant la période périopératoire, pour un suivi d'une durée de 18 mois. À chaque temps de mesure, les participantes remplissent plusieurs questionnaires auto-rapportés, dont le Questionnaire sur les symptômes physiques (qui évalue 19 symptômes somatiques) et le Multidimensional Perfectionism Scale (Frost, Marten, Lahart, \& Rosenblate, 1990) qui inclut l'évaluation des dimensions du perfectionnisme et un questionnaire sociodémographique.

\section{Résultats et discussion}

Les résultats des régressions linéaires multiples (objectif 1) montrent que les préoccupations perfectionnistes sont un prédicteur significatif de l'interférence perçue des symptômes somatiques (analyse transversale; $\beta=0,18, t(411)=2,77$, $p=0,006$ ). Les résultats de l'analyse multi-niveaux (objectif 2 ) montrent que seules les préoccupations perfectionnistes permettent de prédire significativement les points de départ des différentes trajectoires au point de vue de l'interférence perçue (analyse longitudinale; $\beta=0,14, p=0,009$ ). Dans ces analyses, une trajectoire est définie comme une droite de régression qui permet de résumer la relation entre le temps (0-18 mois) et la variable dépendante (symptômes somatiques). Aucune association significative n'est observée entre les aspirations perfectionnistes et les symptômes somatiques de manière transversale et longitudinale.

Les deux résultats révèlent que plus une patiente rapporte des préoccupations perfectionnistes, plus elle perçoit que ses symptômes interfèrent dans sa vie. Il est proposé que la culture du ruban rose, soit un contexte particulier associé au cancer du sein qui met de l'avant l'importance de l'esprit Àcombatif, pourrait contribuer aux préoccupations élevées que ces femmes ont envers l'échec et le jugement des autres (Ehrenreich, 2009; Savard, 2010). Les femmes perfectionnistes qui sont atteintes de ce cancer peuvent ressentir de la pression à garder l'esprit combatif prôné par la culture du ruban rose et être plus susceptibles de s'inquiéter au sujet de leurs symptômes. Sirois et Molnar (2016) rapportent d'ailleurs que les préoccupations perfectionnistes sont associées à différentes formes de persévérations cognitives, telles que la rumination et les inquiétudes en général.

Cette étude améliore la compréhension de la relation entre les dimensions du perfectionnisme et les symptômes somatiques en contexte oncologique. Les résultats suggèrent que certaines interventions ciblant le perfectionnisme pourraient être ajoutées parallèlement à leurs traitements oncologiques pour contribuer à amoindrir les effets délétères possibles du perfectionnisme sur l'interférence perçue des symptômes des femmes atteintes de ce cancer. II serait intéressant de répliquer les résultats de cette étude auprès de patients atteints d'autres types de cancer pour vérifier si des résultats similaires sont observés.

\section{Références}

Ehrenreich, B. (2009). Bright-sided: How positive thinking is undermining America. New York: Metropolitan Books.

Ferreira, K. A., Kimura, M., Teixeira, M. J., Mendoza, T. R., da Nobrega, J. C., Graziani, S. R., \& Takagaki, T. Y. (2008). Impact of cancer-related symptom synergisms on healthrelated quality of life and performance status. Journal of Pain and Symptom Management, 35(6), 604-616. https:// doi.org/10.1016/j.jpainsymman.2007.07.010

Frost, R. O., Marten, P., Lahart, C., \& Rosenblate, R. (1990). The dimensions of perfectionism. Cognitive Therapy and Research, 14(5), 449-468. https://doi.org/10.1007/ bf01172967

Saboonchi, F. \& Lundh, L.-G. (2003). Perfectionism, anger, somatic health, and positive affect. Personality and Individual Differences, 35(7), 1585-1599. https://doi.org/10.1016/ s0191-8869(02)00382-3

Savard, J. (2010). Faire face au cancer - avec la pensée réaliste. Québec: Flammarion Québec.

Savard, J., Ivers, H., Villa, J., Caplette-Gingras, A., \& Morin, C. M. (2011). Natural course of insomnia comorbid with cancer: an 18-month longitudinal study. Journal of Clinical Oncology, 29(26), 3580-3586. https://doi.org/10.1200/ JCO.2010.33.2247 
Savard, J., Villa, J., Ivers, H., Simard, S., \& Morin, C. M. (2009). Prevalence, natural course, and risk factors of insomnia comorbid with cancer over a 2-month period. Journal of Clinical Oncology, 27(31), 5233-5239. https://doi. org/10.1200/JCO.2008.21.6333

Sirois, F. M. et Molnar, D. S. (2016). Perfectionnism, Health and Well-Being. London: Springer.
Société canadienne du cancer. (2020). Vue d'ensemble des statistiques sur le cancer. Repéré à https://www.cancer.ca/ fr-ca/cancer-information/cancer-101/cancer-statistics-at-aglance $/$ ?region $=q c$

White, C. \& Schweitzer, R. (2000). The role of personality in the development and perpetuation of chronic fatigue syndrome. Journal of Psychosomatic Research, 48(6), 515524. https://doi.org/10.1016/s0022-3999(00)00087-8

\title{
Pour citer l'article
}

Séguin-Kaercher, N., Cadorette, J., Massicote, V., Ivers, H., \& Savard, J. (2020). La relation entre le perfectionnisme et les symptômes somatiques chez les femmes atteintes d'un cancer du sein. Psycause: Revue scientifique étudiante de l'École de psychologie de l'Université Laval, 10(2), 15-17.

\section{LE RAPPEL DE RÊVES CHEZ LES AÎNÉS PRÉSENTANT UN TROUBLE COGNITIF LÉGER}

\author{
Florence BELZILE¹, Geneviève SANFAÇON-GAGNON¹, Béatrice SASSEVILLE¹, Mélissa MAILLOUX \& Célyne BASTIEN² \\ ${ }^{1}$ École de Psychologie, Université Laval, ${ }^{2}$ Centre de recherche CERVO, Québec, Canada \\ *florence.belzile-marsolais.1@ulaval.ca
}

\section{Mots-clés : Rappel de rêves, sommeil, trouble cognitif léger, maladie d'Alzheimer}

Chez les aînés, la qualité du sommeil diminue en raison d'une réduction normale et progressive de la durée du temps passé dans le stade du sommeil paradoxal (SP), soit le stade associé à une fréquence de rappel de rêves (FRR) plus élevée. En ce sens, les écrits scientifiques démontrent qu'avec le vieillissement, il existe une diminution de la FRR (Guénolé, Marcaggi, Baleyte, \& Guarma, 2010). Comparativement au vieillissement normal, les individus ayant un trouble cognitif léger de type amnésique (TCL-a) présentent une réduction encore plus marquée du temps passé en SP. Ce trouble, prodrome probable delamaladied'Alzheimer(MA), secaractérise par des difficultés objectives et subjectives de mémoire (Albert et coll., 2011). Avant même l'apparition de déficits cognitifs, un sommeil de mauvaise qualité est susceptible de prédire le développement du TCL-a (Maestri et coll., 2015). Il existe également une relation négative significative entre les déficits mnésiques et la qualité du sommeil chez cette population (Sun et coll., 2016). Actuellement, un manque d'interventions pouvant prévenir l'évolution de ce trouble vers la MA persiste. L'activité physique semble cependant associée à un risque réduit de déclin cognitif pathologique et de développement de la MA (Gasquoine, 2018). Par ailleurs, un programme d'activité physique (PAP) de 20 semaines fut efficace pour améliorer à la fois les fonctions cognitives et la qualité du sommeil des personnes atteintes d'un TCL-a y ayant participé (Bademli, Lok, Canbaz, \& Lok, 2018).
La relation entre les déficits mnésiques et la FRR semble être bien établie dans les écrits scientifiques. II serait alors essentiel d'investiguer si la FRR peut constituer un marqueur de la mémoire susceptible de prédire le déclin cognitif des personnes présentant un TCL-a. II apparaît d'autant plus important d'évaluer l'efficacité potentielle d'un PAP pour augmenter leurs capacités mnésiques, plus spécifiquement leur FRR. Ainsi, le premier objectif est d'investiguer l'efficacité d'un PAP en mesurant son influence sur la FRR des TCL-a et le second objectif est d'étudier l'efficacité d'un PAP en mesurant son influence sur les symptômes d'insomnie.

\section{Méthode}

Quinze individus de 60 ans et plus $(M=74, E T=6,67)$, dont 9 femmes, sont recrutés sur la base de la présence d'un diagnostic de TCL-a (Albert et coll., 2011). Ceux-ci doivent également présenter des difficultés subjectives de sommeil, sans nécessairement satisfaire les critères diagnostiques de I'insomnie, et un niveau faible à modéré d'activité physique au Questionnaire d'activités physiques pour les personnes âgées. Les participants ont complété: 1) le Questionnaire sur la Fréquence de Rappel de Rêves (QFRR), permettant d'évaluer la FRR; et 2) I'Index de Sévérité de I'Insomnie (ISI), évaluant les symptômes subjectifs de l'insomnie. Ensuite, le PAP de 12 semaines a débuté à raison de trois séances hebdomadaires de 60 à 90 minutes. Le QFRR et I'ISI ont été administrés une seconde fois lorsque le PAP fut achevé. 